\title{
LOCAL UNIQUENESS OF SOLUTIONS OF THE CHARACTERISTIC CAUCHY PROBLEM
}

\author{
GERSON PETRONILHO
}

(Received 16 May 1990; revised 13 September 1990)

Communicated by E. N. Dancer

\begin{abstract}
Local uniqueness of solutions of the characteristic Cauchy problem is shown for operators which are perturbations of operators which already have such a uniqueness.
\end{abstract}

1991 Mathematics subject classification (Amer. Math. Soc.): 35 L 15, 35 A 07.

Keywords and phrases: Local uniqueness, characteristic, concatenations, Carleman inequality.

\section{Introduction}

This work is related to discrete phenomena in the local uniqueness of solutions of the characteristic Cauchy problem for operators with double characteristics at a point of the initial curve.

On this type of phenomena F. Treves [5] furnished an example, that is, the Cauchy problem for the equation

$$
\left(\partial_{t}+t \partial_{x}\right)\left(\partial_{t}-t \partial_{x}\right) u-c \partial_{x} u=0
$$

with data given in $x=0$, and proved that for the problem in the upper halfplane $x>0$ there is uniqueness of solutions, if and only if $c \neq 0,2,4, \ldots$.

A. P. Bergamasco and H. S. Ribeiro [1] and A. Menikoff [3], extended this study for operators of the form

$$
\left(\partial_{t}+a t^{k} \partial_{x}\right)\left(\partial_{t}-b t^{k} \partial_{x}\right)-c t^{k-1} \partial_{x}
$$

The author was partially supported by CNPq (Brazil).

(C) 1992 Australian Mathematical Society 0263-6115/92 \$A2.00+0.00 
where $k$ is an odd natural number. In [1], $a=b<0$ and $c \in \mathbb{R}$, while in [3], $a, b>0$ and $c \in \mathbb{C}$.

We extend for operators of the form

$$
\begin{aligned}
P\left(c_{1}, c_{2}\right)= & \left(\partial_{t}+a_{1} t^{k} \partial_{x}+a_{2} t^{k}\right)\left(\partial_{t}-b_{1} t^{k} \partial_{x}-b_{2} t^{k}\right) \\
& -c_{1} t^{k-1} \partial_{x}-c_{2} t^{k-1}
\end{aligned}
$$

where $k$ is an odd natural number, $c_{1}, c_{2} \in \mathbb{C}, a_{1}, b_{1}>0$, and $a_{2}, b_{2} \leq 0$.

In Section 2 we prove, under suitable assumptions, the local uniqueness, in the class $C^{3}$, of solutions of the characteristic Cauchy problem for the operators $P\left(c_{1}, c_{2}\right)$. The essential point in the proof is getting an appropriate Carleman inequality.

In Section 3, by using the results of Section 2 and a variation of concatenations in O. R. B. Oliveira [4], we prove that there is an integer $m$ depending only on $\operatorname{Re}\left(c_{1}\right)$ such that the local uniqueness of solutions in the class $C^{m}$ holds in the characteristic Cauchy problem for the operators $P\left(c_{1}, c_{2}\right)$ if $\operatorname{Re}\left(c_{1}\right) \neq j(k+1) \delta_{1}, \operatorname{Re}\left(c_{1}\right) \neq j(k+1) \delta_{1}+\delta_{1}, j=0,1,2, \ldots$ and $\delta_{1}=a_{1}+b_{1}$.

If $a_{2}=b_{2}=c_{2}=0$ then $P\left(c_{1}, c_{2}\right)$ is the operator studied in [3].

We show also that the uniqueness for the operators $P\left(c_{1}, c_{2}\right)$ holds (when $a_{1}, b_{1}<0$ and $\left.a_{2}, b_{2} \geq 0\right)$ if $\operatorname{Re}\left(c_{1}\right) \neq-\delta_{1}[k+j(k+1)], \operatorname{Re}\left(c_{1}\right) \neq-\delta_{1}(j+$ $1)(k+1)$. This result contains part of [1, Theorem 3.1].

We observe also that the local uniqueness of solutions in the class of distributions holds for these operators (see [1] and B. Birkeland and J. Persson [2]).

\section{A Carleman inequality and uniqueness}

In this section, we prove a Carleman inequality which is needed in order to prove local uniqueness of solutions of the characteristic Cauchy problem for operators of the form

$$
P=P\left(c_{1}, c_{2}\right)=X Y-c_{1} t^{k-1} \partial_{x}-c_{2} t^{k-1}
$$

where

$$
\begin{aligned}
& X=\partial_{t}+a_{1} t^{k} \partial_{x}+a_{2} t^{k}, \\
& Y=\partial_{t}-b_{1} t^{k} \partial_{x}-b_{2} t^{k}, \\
& a_{1}, b_{1}>0, a_{2}, b_{2} \leq 0,
\end{aligned}
$$

$c_{1}, c_{2}$ are complex numbers, $k$ is an odd natural number and $(t, x) \in$ $[-T, T] \times \mathbb{R}, T>0$. 
Since

$$
\begin{aligned}
P\left(c_{1}, c_{2}\right)= & \partial_{t}^{2}-a_{1} b_{1} t^{2 k} \partial_{x}^{2}+\left(a_{1}-b_{1}\right) t^{k} \partial_{t} \partial_{x}-\left(c_{1}+b_{1} k\right) t^{k-1} \partial_{x} \\
& -\left(a_{1} b_{2}+a_{2} b_{1}\right) t^{2 k} \partial_{x}-\left(c_{2}+b_{2} k\right) t^{k-1}-a_{2} b_{2} t^{2 k} \\
& +\left(a_{2}-b_{2}\right) t^{k} \partial_{t},
\end{aligned}
$$

if

$$
f(t)=-\frac{1}{2} \int_{0}^{t}\left(a_{2}-b_{2}\right) s^{k} d s
$$

and

$$
u(t, x)=\exp [f(t)] \cdot v(t, x)
$$

we obtain

$$
P\left(c_{1}, c_{2}\right) u=\exp [f(t)] \cdot Q\left(c_{1}, c_{2}\right) v
$$

where

$$
\begin{aligned}
Q= & Q\left(c_{1}, c_{2}\right)=\partial_{t}^{2}-a_{1} b_{1} t^{2 k} \partial_{x}^{2}+\left(a_{1}-b_{1}\right) t^{k} \partial_{t} \partial_{x}-\left(c_{1}+b_{1} k\right) t^{k-1} \partial_{x} \\
& -\frac{1}{2}\left(a_{1}+b_{1}\right)\left(a_{2}+b_{2}\right) t^{2 k} \partial_{x}-\left[c_{2}+\frac{k}{2}\left(a_{2}+b_{2}\right)\right] t^{k-1} \\
& -\frac{1}{4}\left(a_{2}+b_{2}\right)^{2} t^{2 k}
\end{aligned}
$$

The purpose of introducing the integrating factor $\exp [f(t)]$ was to obtain a new operator, $Q$, in which the term $\left(a_{2}-b_{2}\right) t^{k} \partial_{t}$ is not present.

By using the formula (2.2), we can see that the local uniqueness of solutions of the characteristic Cauchy problem holds for $P$ if and only if it holds for $Q$.

LEMMA 2.1. Let $Q\left(c_{1}, c_{2}\right)$ be given by (2.3). If $\operatorname{Re}\left[c_{1}+(k / 2)\left(a_{1}+b_{1}\right)\right] \leq$ $0, \operatorname{Re}\left[c_{2}+(k / 2)\left(a_{2}+b_{2}\right)\right] \geq 0$ and $Q_{\tau}$ is the operator

$$
Q_{\tau}=\exp (\tau x) \cdot Q \cdot \exp (-\tau x)
$$

then the following inequalities hold for all $v \in C_{c}^{3}(\mathbb{O} ; \mathbb{R})$ and for $\tau \geq 1$, where $\mathbb{O} \subset\left\{(t, x) \in \mathbb{R}^{2}:|t| \leq T\right\}$ is a nonempty bounded open subset of $\mathbb{R}^{2}$.

$$
\text { I-1: } \quad \operatorname{Re}<Q_{\tau} v,\left(\partial_{x}-\tau\right) v>\geq a_{1} b_{1} \iint_{0} t^{2 k}|v|^{2} d t d x
$$

where $\langle$,$\rangle is the usual inner product of \mathbb{L}^{2}(\mathbb{O} ; \mathbb{R})$.

I-2 : $\quad \iint_{0} a_{1} b_{1} t^{2 k} \exp (2 \tau x)|v|^{2} d t d x \leq \iint_{0} \exp (2 \tau x)\left|Q v \cdot \partial_{x} v\right| d t d x$. 
Proof. Since

$$
\begin{aligned}
Q_{\tau} v= & \partial_{t}^{2} v-a_{1} b_{1} t^{2 k}\left(\partial_{x}-\tau\right)^{2}+\left(a_{1}-b_{1}\right) t^{k} \partial_{t}\left(\partial_{x}-\tau\right)-\left(c_{1}+b_{1} k\right) t^{k-1}\left(\partial_{x}-\tau\right) \\
& -\frac{1}{2}\left(a_{1}+b_{1}\right)\left(a_{2}+b_{2}\right) t^{2 k}\left(\partial_{x}-\tau\right)-\left[c_{2}+\frac{k}{2}\left(a_{2}+b_{2}\right)\right] t^{k-1} \\
& -\frac{1}{4}\left(a_{2}+b_{2}\right)^{2} t^{2 k}
\end{aligned}
$$

integrations by parts show that

$$
\begin{aligned}
\operatorname{Re}\left\langle Q_{\tau} v,\left(\partial_{x}-\tau\right) v\right\rangle= & \tau \iint_{\mathbf{0}}\left|\partial_{t} v\right|^{2} d t d x+a_{1} b_{1} \tau \iint_{0} t^{2 k}\left(\left|\partial_{x} v\right|^{2}+\tau^{2}|v|^{2}\right) d t d x \\
& -\operatorname{Re}\left[c_{1}+\frac{k}{2}\left(a_{1}+b_{1}\right)\right] \iint_{0} t^{k-1}\left(\left|\partial_{x} v\right|^{2}+\tau^{2}|v|^{2}\right) d t d x \\
& -\frac{1}{2}\left(a_{1}+b_{1}\right)\left(a_{2}+b_{2}\right) \iint_{0} t^{2 k}\left(\left|\partial_{x} v\right|^{2}+\tau^{2}|v|^{2}\right) d t d x \\
& +\operatorname{Re}\left[c_{2}+\frac{k}{2}\left(a_{2}+b_{2}\right)\right] \tau \iint_{0} t^{k-1}|v|^{2} d t d x \\
& +\frac{1}{4}\left(a_{2}+b_{2}\right)^{2} \tau \iint_{0} t^{2 k}|v|^{2} d t d x \\
\geq & a_{1} b_{1} \iint_{0} t^{2 k}|v|^{2} d t d x .
\end{aligned}
$$

The proof of $I-1$ is complete.

We prove $I-2$, as follows:

$$
\begin{aligned}
\iint_{\mathbb{0}} a_{1} b_{1} t^{2 k} \exp (2 \tau x)|v|^{2} d t d x & \leq \operatorname{Re}\left\langle Q_{\tau}[\exp (\tau x) v],\left(\partial_{x}-\tau\right)[\exp (\tau x) v]\right\rangle \\
& =\operatorname{Re}\left\langle\exp (\tau x) Q v, \exp (\tau x) \partial_{x} v\right\rangle \\
& \leq \iint_{0} \exp (2 \tau x)\left|Q v \cdot \partial_{x} v\right| d t d x
\end{aligned}
$$

By using inequality $\mathrm{I}-2$ and following the proof of [6, Theorem 2.3], we can prove

THEOREM 2.1. Let $Q$ be given by (2.3) and assume that

$$
\operatorname{Re}\left[c_{1}+(k / 2)\left(a_{1}+b_{1}\right)\right] \leq 0
$$

and

$$
\operatorname{Re}\left[c_{2}+(k / 2)\left(a_{2}+b_{2}\right)\right] \geq 0 .
$$

Let $\mathbb{F} \subset \mathbb{O}$ be a closed subset such that

$$
\mathbb{K}=\mathbb{F} \cap\{(t, x) \in \mathbb{O}: x \geq 0\}
$$


is compact. Then there is an open neighborhood $\mathbb{U}$ of $\mathbb{K}$ such that any function $u \in C^{3}(\mathbb{O} ; \mathbb{R})$ satisfying

$$
Q u=0 \text { in } \mathbb{O} ; \operatorname{supp} u \subset \mathbb{F}
$$

must vanish in $\mathbb{U}$.

\section{Concatenation and uniqueness}

Theorem 2.1 guarantees local uniqueness for the operator $Q\left(c_{1}, c_{2}\right)$ and therefore for $P\left(c_{1}, c_{2}\right)$ if $\operatorname{Re}\left(c_{1}+(k / 2) \delta_{1}\right) \leq 0$ and $\operatorname{Re}\left(c_{2}+(k / 2) \delta_{2}\right) \geq 0$, where $\delta_{1}=a_{1}+b_{1}$ and $\delta_{2}=a_{2}+b_{2}$, that is, if $\operatorname{Re}\left(c_{1}\right)$ is small and $\operatorname{Re}\left(c_{2}\right)$ is large.

To prove uniqueness when $\operatorname{Re}\left(c_{1}\right)$ is large and any $c_{2}$, we shall use the method of concatenations.

Note that we have

$$
\begin{gathered}
{[X, Y]=-k t^{k-1}\left(\delta_{1} \partial_{x}+\delta_{2}\right)} \\
{\left[t\left(\delta_{1} \partial_{x}+\delta_{2}\right), Y X\right]=-\left(\delta_{1} \partial_{x}+\delta_{2}\right)(X+Y)} \\
{\left[t\left(\delta_{1} \partial_{x}+\delta_{2}\right) Y, t^{k-1}\left(c_{1} \partial_{x}+c_{2}\right)\right]} \\
=(k-1) t^{k-1}\left(\delta_{1} \partial_{x}+\delta_{2}\right)\left(c_{1} \partial_{x}+c_{2}\right)
\end{gathered}
$$

Since $Y=X-t^{k}\left(\delta_{1} \partial_{x}+\delta_{2}\right)$, we have

$$
Y^{2}=X Y-t^{k}\left(\delta_{1} \partial_{x}+\delta_{2}\right) Y .
$$

We shall try to find operators

$$
T=t\left(\delta_{1} \partial_{x}+\delta_{2}\right) Y+\left(A_{1} \partial_{x}+B_{1}\right), \quad S=t\left(\delta_{1} \partial_{x}+\delta_{2}\right) Y+\left(A_{2} \partial_{x}+B_{2}\right)
$$

so that

$$
T P\left(c_{1}, c_{2}\right)=P\left(c_{1}^{\prime}, c_{2}^{\prime}\right) S
$$


By using (3.1)-(3.4) we have

$$
\begin{aligned}
& T P\left(c_{1}, c_{2}\right)=\left[t\left(\delta_{1} \partial_{x}+\delta_{2}\right) Y+\left(A_{1} \partial_{x}+B_{1}\right)\right]\left(X Y-c_{1} t^{k-1} \partial_{x}-c_{2} t^{k-1}\right) \\
& =t\left(\delta_{1} \partial_{x}+\delta_{2}\right) Y X Y-t\left(\delta_{1} \partial_{x}+\delta_{2}\right) Y t^{k-1}\left(c_{1} \partial_{x}+c_{2}\right) \\
& +\left(A_{1} \partial_{x}+B_{1}\right) X Y-\left(A_{1} \partial_{x}+B_{1}\right) t^{k-1}\left(c_{1} \partial_{x}+c_{2}\right) \\
& =-\left(\delta_{1} \partial_{x}+\delta_{2}\right) X Y-\left(\delta_{1} \partial_{x}+\delta_{2}\right) Y^{2}+Y X t\left(\delta_{1} \partial_{x}+\delta_{2}\right) Y \\
& -\left[t\left(\delta_{1} \partial_{x}+\delta_{2}\right) Y, t^{k-1}\left(c_{1} \partial_{x}+c_{2}\right)\right] \\
& -t^{k-1}\left(c_{1} \partial_{x}+c_{2}\right) t\left(\delta_{1} \partial_{x}+\delta_{2}\right) Y \\
& +\left(A_{1} \partial_{x}+B_{1}\right) X Y-\left(A_{1} \partial_{x}+B_{1}\right) t^{k-1}\left(c_{1} \partial_{x}+c_{2}\right) \\
& =\left[-2\left(\delta_{1} \partial_{x}+\delta_{2}\right)+\left(A_{1} \partial_{x}+B_{1}\right)\right] X Y+\left(\delta_{1} \partial_{x}+\delta_{2}\right) t^{k}\left(\delta_{1} \partial_{x}+\delta_{2}\right) Y \\
& +(X Y-[X, Y]) t\left(\delta_{1} \partial_{x}+\delta_{2}\right) Y \\
& -(k-1) t^{k-1}\left(\delta_{1} \partial_{x}+\delta_{2}\right)\left(c_{1} \partial_{x}+c_{2}\right) \\
& -t^{k-1}\left(c_{1} \partial_{x}+c_{2}\right) t\left(\delta_{1} \partial_{x}+\delta_{2}\right) Y-\left(A_{1} \partial_{x}+B_{1}\right) t^{k-1}\left(c_{1} \partial_{x}+c_{2}\right) \\
& =X Y t\left(\delta_{1} \partial_{x}+\delta_{2}\right) Y+k t^{k-1}\left(\delta_{1} \partial_{x}+\delta_{2}\right) t\left(\delta_{1} \partial_{x}+\delta_{2}\right) Y \\
& -\left[-2\left(\delta_{1} \partial_{x}+\delta_{2}\right)+\left(A_{1} \partial_{x}+B_{1}\right)\right] X Y \\
& +t^{k-1}\left(\delta_{1} \partial_{x}+\delta_{2}\right) t\left(\delta_{1} \partial_{x}+\delta_{2}\right) Y \\
& -(k-1) t^{k-1}\left(\delta_{1} \partial_{x}+\delta_{2}\right)\left(c_{1} \partial_{x}+c_{2}\right) \\
& -t^{k-1}\left(c_{1} \partial_{x}+c_{2}\right) t\left(\delta_{1} \partial_{x}+\delta_{2}\right) Y-\left(A_{1} \partial_{x}+B_{1}\right) t^{k-1}\left(c_{1} \partial_{x}+c_{2}\right) \\
& =\left\{X Y-t^{k-1}\left[\left(c_{1} \partial_{x}+c_{2}\right)-(k-1)\left(\delta_{1} \partial_{x}+\delta_{2}\right)\right]\right\} \\
& \circ\left[t\left(\delta_{1} \partial_{x}+\delta_{2}\right) Y-2\left(\delta_{1} \partial_{x}+\delta_{2}\right)+\left(A_{1} \partial_{x}+B_{1}\right)\right] \\
& +t^{k-1}\left[\left(c_{1} \partial_{x}+c_{2}\right)-(k+1)\left(\delta_{1} \partial_{x}+\delta_{2}\right)\right] \\
& -\left[-2\left(\delta_{1} \partial_{x}+\delta_{2}\right)+\left(A_{1} \partial_{x}+B_{1}\right)\right] \\
& -(k-1) t^{k-1}\left(\delta_{1} \partial_{x}+\delta_{2}\right)\left(c_{1} \partial_{x}+c_{2}\right)-\left(A_{1} \partial_{x} B_{1}\right) t^{k-1}\left(c_{1} \partial_{x}+c_{2}\right) \text {. }
\end{aligned}
$$

If we choose $A_{1}=2 \delta_{1}-c_{1}$ and $B_{1}=2 \delta_{2}-c_{2}$, we obtain (3.5), that is,

$$
\begin{aligned}
{\left[t\left(\delta_{1} \partial_{x}+\delta_{2}\right) Y+2\left(\delta_{1} \partial_{x}+\delta_{2}\right)-\left(c_{1} \partial_{x}+c_{2}\right)\right]\left[X Y-t^{k-1}\left(c_{1} \partial_{x}+c_{2}\right)\right] } \\
=\left\{X Y-t^{k-1}\left[\left(c_{1} \partial_{x}+c_{2}\right)-(k+1)\left(\delta_{1} \partial_{x}+\delta_{2}\right)\right]\right\} \\
\quad \circ\left[t\left(\delta_{1} \partial_{x}+\delta_{2}\right) Y-\left(c_{1} \partial_{x}+c_{2}\right)\right]
\end{aligned}
$$

Now, we shall try to find operators $Q$ and $R$ so that

$$
Q S+R P\left(c_{1}, c_{2}\right)=\left(c_{1} \partial_{x}+c_{2}\right)\left[\left(\delta_{1} \partial_{x}+\delta_{2}\right)-\left(c_{1} \partial_{x}+c_{2}\right)\right]
$$


To obtain (3.6) write

$$
\begin{aligned}
X S & =X\left[t\left(\delta_{1} \partial_{x}+\delta_{2}\right) Y-\left(c_{1} \partial_{x}+c_{2}\right)\right]=X t\left(\delta_{1} \partial_{x}+\delta_{2}\right) Y-X\left(c_{1} \partial_{x}+c_{2}\right) \\
& =\left(\delta_{1} \partial_{x}+\delta_{2}\right) Y+t\left(\delta_{1} \partial_{x}+\delta_{2}\right) X Y-\left(c_{1} \partial_{x}+c_{2}\right) X .
\end{aligned}
$$

Then

$$
\begin{aligned}
X S- & t\left(\delta_{1} \partial_{x}+\delta_{2}\right) P\left(c_{1}, c_{2}\right) \\
& =X S-t\left(\delta_{1} \partial_{x}+\delta_{2}\right) X Y+t\left(\delta_{1} \partial_{x}+\delta_{2}\right) t^{k-1}\left(c_{1} \partial_{x}+c_{2}\right) \\
& =\left(\delta_{1} \partial_{x}+\delta_{2}\right) Y-\left(c_{1} \partial_{x}+c_{2}\right) X+t^{k}\left(\delta_{1} \partial_{x}+\delta_{2}\right)\left(c_{1} \partial_{x}+c_{2}\right)
\end{aligned}
$$

Since $X-Y=t^{k}\left(\delta_{1} \partial_{x}+\delta_{2}\right)$ we have

$$
X S-t\left(\delta_{1} \partial_{x}+\delta_{2}\right) P\left(c_{1}, c_{2}\right)=\left[\left(\delta_{1} \partial_{x}+\delta_{2}\right)-\left(c_{1} \partial_{x}+c_{2}\right)\right] Y
$$

and therefore

$$
\begin{aligned}
& {\left[t\left(\delta_{1} \partial_{x}+\delta_{2}\right) X+\left(c_{1} \partial_{x}+c_{2}\right)-\left(\delta_{1} \partial_{x}+\delta_{2}\right)\right] S-t^{2}\left(\delta_{1} \partial_{x}+\delta_{2}\right)^{2} P\left(c_{1}, c_{2}\right)} \\
& \quad=\left(c_{1} \partial_{x}+c_{2}\right)\left[\left(\delta_{1} \partial_{x}+\delta_{2}\right)-\left(c_{1} \partial_{x}+c_{2}\right)\right] .
\end{aligned}
$$

Then (3.6) holds with

$$
Q=t\left(\delta_{1} \partial_{x}+\delta_{2}\right) X+\left(c_{1} \partial_{x}+c_{2}\right)-\left(\delta_{1} \partial_{x}+\delta_{2}\right)
$$

and

$$
R=-t^{2}\left(\delta_{1} \partial_{x}+\delta_{2}\right)^{2} \text {. }
$$

The computations above yield the following result.

LEMMA 3.1. For $j, l=0,1,2, \ldots$, let

$$
\begin{aligned}
c_{1 j}= & c_{1}-j(k+1) \delta_{1}, \quad c_{2 l}=c_{2}-l(k+1) \delta_{2} \\
T_{j, l}= & t\left(\delta_{1} \partial_{x}+\delta_{2}\right) Y+2\left(\delta_{1} \partial_{x}+\delta_{2}\right)-\left(c_{1 j} \partial_{x}+c_{2 l}\right) \\
& S_{j, l}=t\left(\delta_{1} \partial_{x}+\delta_{2}\right) Y-\left(c_{1 j} \partial_{x}+c_{2 l}\right) \\
& P\left(c_{1 j}, c_{21}\right)=X Y-t^{k-1}\left(c_{1 j} \partial_{x}+c_{2 l}\right) \\
Q_{j, l}= & t\left(\delta_{1} \partial_{x}+\delta_{2}\right) X+\left(c_{1 j} \partial_{x}+c_{2 l}\right)-\left(\delta_{1} \partial_{x}+\delta_{2}\right) .
\end{aligned}
$$

Then

$$
T_{j, l} P\left(c_{1 j}, c_{2 l}\right)=P\left(c_{1, j+1}, c_{2, l+1}\right) S_{j, l}
$$

and we can find operators $Q_{j, l}$ such that

$$
Q_{j, l} S_{j, l}+R P\left(c_{1 j}, c_{2 l}\right)=\left(c_{1 j} \partial_{x}+c_{2 l}\right)\left[\left(\delta_{1} \partial_{x}+\delta_{2}\right)-\left(c_{1 j} \partial_{x}+c_{2 l}\right)\right] \text {. }
$$

Consider the statement,

$\mathbf{A}_{j, l}^{m}$ : Every $u \in C^{m}(\mathbb{O})$ which satisfies $P\left(c_{1 j}, c_{2 l}\right) u=0$ in $\mathbb{O}$ with supp $u \subset$ $\mathbb{F}$ must vanish in $\Omega$, where $\Omega$ is an open neighborhood of $\mathbb{K}$. (Here, $\mathbb{O}, \mathbb{F}$ and $\mathbb{K}$ are as in Theorem 2.1 and $m \geq 3$ ). 
LEMMA 3.2. If $c_{1 j} \neq 0$ and $c_{1 j} \neq \delta_{1}$ then $\mathbb{A}_{j+1, l+1}^{m}$ implies $\mathbf{A}_{j, l}^{m+2}$. Since

Proof. Let $u \in C^{m+2}(\mathbb{O})$ so that $P\left(c_{1 j}, c_{2 l}\right)=0$ in $\mathbb{O}$ and supp $u \subset \mathbb{F}$.

$$
T_{j, l} P\left(c_{1 j}, c_{2 l}\right)=P\left(c_{1, j+1}, c_{2, l+1}\right) S_{j, l}(\text { see }(3.7))
$$

we have

$$
P\left(c_{1, j+1}, c_{2, l+1}\right)\left(S_{j, l} u\right)=0 \text { in } \mathbb{0} .
$$

Since $S_{j, l}$ is a second order linear operator we have $S_{j, l} u \in C^{m}(\mathbb{O})$ and therefore by hypothesis

$$
S_{j, l} u=0 \text { in } \Omega \text {. }
$$

By (3.8) there are operators $Q_{j, l}$ and $R$ such that

$$
Q_{j, l} S_{j, l} u+R P\left(c_{1 j}, c_{2 l}\right) u=\left(c_{1 j} \partial_{x}+c_{2 l}\right)\left[\left(\delta_{1}-c_{1 j}\right) \partial_{x}+\left(\delta_{2}-c_{2 l}\right)\right] u
$$

and therefore

$$
\left(c_{1 j} \partial_{x}+c_{2 l}\right)\left[\left(\delta_{1}-c_{1 j}\right) \partial_{x}+\left(\delta_{2}-c_{2 l}\right)\right] u=0 \text { in } \Omega .
$$

Since $c_{1 j} \neq 0$ we have local uniqueness of solutions of the noncharacteristic Cauchy problem for the operator $c_{1 j} \partial_{x}+c_{2 l}$ and therefore

$$
\left[\left(\delta_{1}-c_{1 j}\right) \partial_{x}+\left(\delta_{2}-c_{2 l}\right)\right] u=0 \text { in } \mathbf{W},
$$

where $\mathbf{W}$ is an open neighborhood of $\mathbb{K}$.

Now, since by the hypothesis $c_{1 j} \neq \delta_{1}$, the uniqueness for the operator $\left(\delta_{1}-c_{1 j}\right) \partial_{x}+\left(\delta_{2}-c_{2 l}\right)$ implies $u=0$ in $\mathbb{U}$, where $\mathbb{U}$ is an open neighborhood of $\mathbb{K}$.

THEOREM 3.1. Suppose that $c_{1} \neq j(k+1) \delta_{1}$ and $c_{1} \neq j(k+1) \delta_{1}+\delta_{1}$, $j=0,1,2, \ldots$ and $c_{2} \in \mathbb{C}$. Let $\mathbb{O}, \mathbb{F}$ and $\mathbb{K}$ be as in Theorem 2.1. Then there is an integer $m$ depending only on $\operatorname{Re}\left(c_{1}\right)$ and an open neighborhood $\mathbb{U}$ of $\mathbb{K}$ such that every $u \in C^{m}(\mathbb{O}, \mathbb{R})$ with support in $\mathbb{F}$ which satisfies

$$
P\left(c_{1}, c_{2}\right) u=0 \text { in } \mathbb{O}
$$

must vanish in $\mathbb{U}$.

Proof. Let $c_{1}, c_{2} \in \mathbb{C}$ and $c_{1}^{\prime}=c_{1}+(k / 2) \delta_{1}, c_{2}^{\prime}=c_{2}+(k / 2) \delta_{2}$.

If $\operatorname{Re}\left(c_{1}^{\prime}\right) \leq 0$ and $\operatorname{Re}\left(c_{2}^{\prime}\right) \geq 0$ then Theorem 2.1 guarantees for the operator $Q\left(c_{1}, c_{2}\right)$, and therefore for $P\left(c_{1}, c_{2}\right)$, the uniqueness result stated in Theorem 3.1 with $m=3$.

If $\operatorname{Re}\left(c_{1}^{\prime}\right) \leq 0$ and $\operatorname{Re}\left(c_{2}^{\prime}\right)<0$, let $l_{0}$ be the smallest natural number such that $\operatorname{Re}\left[c_{2 l}+(k / 2) \delta_{2}\right] \geq 0$ where $c_{2 l_{0}}=c_{2}-l_{0}(k+1) \delta_{2}$. Thus, 
$\operatorname{Re}\left[c_{1 l_{0}}+(k / 2) \delta_{1}\right]<0$ where $c_{1 l_{0}}=c_{1}-l_{0}(k+1) \delta_{1}$ and therefore as in the preceding case, $\mathbf{A}_{l_{0}, l_{0}}^{3}$ holds.

We apply Lemma $3.2 l_{0}$ times and conclude that $\mathbb{A}_{0,0}^{3+2 l_{0}}$ holds; in this case $m=3+2 l_{0}$.

If $\operatorname{Re}\left(c_{1}^{\prime}\right)>0$ and $\operatorname{Re}\left(c_{2}^{\prime}\right) \leq 0$, let $j_{0}$ and $l_{0}$ be the smallest natural numbers such that $\operatorname{Re}\left[c_{1 j_{0}}+(k / 2) \delta_{1}\right] \leq 0$ and $\operatorname{Re}\left[c_{2 l_{0}}+(k / 2) \delta_{2}\right] \geq 0$.

If $j_{0}<l_{0}$ we have $\operatorname{Re}\left[c_{1 l_{0}}+(k / 2) \delta_{1}\right] \leq 0$ and therefore as in the first case, $\mathbf{A}_{l_{0}, l_{0}}^{3}$ holds. We apply Lemma 3.2, $l_{0}$ times and conclude that $\mathbb{A}_{0,0}^{3+2 l_{0}}$ holds; in this case $m=3+2 l_{0}$.

If $j_{0}>l_{0}$ we have $\operatorname{Re}\left[c_{2 j_{0}}+(k / 2) \delta_{2}\right] \geq 0$ and as in the preceding case, $\mathrm{A}_{0,0}^{3+2 j_{0}}$ holds; in this case $m=3+2 j_{0}$.

If $\operatorname{Re}\left(c_{1}^{\prime}\right)>0$ and $\operatorname{Re}\left(c_{2}^{\prime}\right)>0$, let $j_{0}$ be the smallest natural number such that $\operatorname{Re}\left[c_{1 j_{0}}+(k / 2) \delta_{1}\right] \leq 0$. Thus, $\operatorname{Re}\left[c_{2 j_{0}}+(k / 2) \delta_{2}\right]>0$ and therefore as in the preceding case, $\mathbb{A}_{0,0}^{3+2 j_{0}}$ holds; in this case $m=3+2 j_{0}$. The proof is complete.

Remark 3.1. Now consider the operator $P\left(c_{1}, c_{2}\right)$ with $a_{1}, b_{1}<0$ and $a_{2}, b_{2} \geq 0$. We can write

$$
\begin{aligned}
P\left(c_{1}, c_{2}\right)= & \left(\partial_{t}+a_{1} t^{k} \partial_{x}+a_{2} t^{k}\right)\left(\partial_{t}-b_{1} t^{k} \partial_{x}-b_{2} t^{k}\right)-c_{1} t^{k-1} \partial_{x}-c_{2} t^{k-1} \\
= & {\left[\partial_{t}+\left(-b_{1}\right) t^{k} \partial_{x}+\left(-b_{2}\right) t^{k}\right]\left[\partial_{t}-\left(-a_{1}\right) t^{k} \partial_{x}-\left(-a_{2}\right) t^{k}\right] } \\
& -\left(c_{1}+k \delta_{1}\right) t^{k-1} \partial_{x}-\left(c_{2}+k \delta_{2}\right) t^{k-1} .
\end{aligned}
$$

If $\operatorname{Re}\left(c_{1}\right) \neq-\delta_{1}[k+j(k+1)]$ and $\operatorname{Re}\left(c_{1}\right) \neq-\delta_{1}(j+1)(k+1), j=$ $0,1,2, \ldots$, then Theorem 3.1 ensures local uniqueness for the operator $P\left(c_{1}, c_{2}\right)$.

In the case that $a_{2}=b_{2}=c_{2}=0$ and $b_{1}=a_{1}$ we obtain part of [1, Theorem 3.1].

REMARK 3.2. As the operator $P\left(c_{1}, c_{2}\right)$ belongs to the class of operators considered in [1, Theorem 4.1], this ensures its uniqueness in the class of distributions.

\section{References}

[1] A. P. Bergamasco and H. S. Ribeiro, 'Uniqueness in a doubly characteristic Cauchy problem', Pacific J. Math. 136 (1989), 229-240.

[2] B. Birkeland and J. Persson, 'The local Cauchy problem in $\mathbb{R}^{2}$ at a point where two characteristic curves have a common tangent', J. Differential Equations 30 (1978), 6488. 
[3] A. Menikoff, 'Uniqueness of the Cauchy problem for a class of partial differential equations with double characteristics', Indiana Univ. Math. J. 25 (1976), 1-21.

[4] O. R. B. de Oliveira, 'Estudo de uma Classe de Operadores Diferenciais Parciais Lineares que se degeneram sobre uma Reta e têm Característica Múltipla fora dela' Ms. C., Instituto de Matemática e Estatística da Universidade de São Paulo, 1989.

[5] F. Treves, 'Discrete phenomena in uniqueness in the Cauchy problem', Proc. Amer. Math. Soc. 46 (1974), 229-233.

[6] F. Treves, Linear partial differential equations with constant coefficients: existence, approximation and regularity of solutions, (Math. and its Applications Vol. 6, Gordon and Breach, New York, 1966).

Universidade Federal de São Carlos (UFSCar)

Departamento de Matemática

Caixa Postal 676

13560 - São Carlos - SP - Brazil 